Poster (029)

Epithelial Ovarian Cancer including Borderline Tumor

https://doi.org/10.3802/jgo.2021.32.S1.029

\section{Clinical outcomes of immunohistochemistry of p53 staining pattern in high-grade serous ovarian carcinoma}

Panarat Orachum, Pilaiwan Kleepkaow, Bundit Chumworathayi, Sanguanchoke Luanratanakorn, Apiwat Aueangkul, Yuwadee Itthirat, Amornrat Temtanakitpaisan*

Department of Obstetrics and Gynecology, Faculty of Medicine, Khon Kaen University, Khon Kaen, Thailand (kunglekmed@hotmail.com)

Objective: To investigate the prevalence of p53 mutations and the associated factors between the immunohistochemistry (IHC) of p53 staining pattern among patients with high-grade serous ovarian carcinoma (HGSOC).

Methods: The study was a retrospective study and enrolled a total of 62 patients with HGSOC, who underwent surgery in Srinagarind Hospital, between January 2016 and December 2020. Histological examination was reveal based on the combination of morphology and IHC staining with p53. The p53 immunostaining pattern was interpreted as a missense mutation, nonsense mutation, or wild-type pattern. Missense (p53 overexpression pattern) and nonsense mutation (null expression p53 pattern) were considered to be $\mathrm{p} 53$ mutation. A wide-type pattern was defined as p53 non-mutation.

Results: The p53 mutation was identified in $93.55 \%$ of patients. When subgroup analysis in p53 mutation group between overexpression $\mathrm{p} 53$ pattern and null expression $\mathrm{p} 53$ pattern in terms of clinicopathological characteristic and initial treatment was performed. Patients with overexpression p53 pattern had more omental metastasis compared to those with null expression p53 pattern $(90.0 \%$ vs. $64.7 \%, \mathrm{p}=0.042)$. There were no statistically significant differences in terms of progression free survival (PFS) (9 months vs. 10 months, $\mathrm{p}=0.813$ ) and overall survival (OS) (12 months vs. 17 months, $\mathrm{p}=0.526$ ) were observed between the 2 groups. Conclusion: The prevalence of $\mathrm{p} 53$ mutations in HGSOC in this study was $93 \%$. Omental metastasis is the one significant pathological factor for predicting an overexpression p53 pattern of HGSOC. However, IHC of p53 staining pattern did not impact on OS and PFS among patients with HGSOC.

Poster (030)

Epithelial Ovarian Cancer including Borderline Tumor https://doi.org/10.3802/jgo.2021.32.\$1.030

\section{Efficacy analysis of niraparib using patient- derived xenografts of rare subtypes of ovarian cancer}

Hiroyuki Kurosu, ${ }^{1, *}$ Hiroshi Asano, ${ }^{1}$ Kanako Hatanaka, ${ }^{2}$ Yoshihiro Matsuno, ${ }^{3}$ Yutaka Hatanaka, ${ }^{2}$ Hidemichi Watari ${ }^{1}$

'Department of Obstetrics and Gynecology, Hokkaido University, Graduate School of Medicine and Faculty of Medicine, Sapporo, Japan (krshryk@gmail.com)

${ }^{2}$ Center for Development of Advanced Diagnostics, Hokkaido University Hospital, Research Division of Genome Companion Diagnostics, Hokkaido University Hospital, Sapporo, Japan

${ }^{3}$ Department of Surgical Pathology, Hokkaido University Hospital, Sapporo, Japan

Objective: Niraparib, a PARP inhibitor, shows an antitumor effect by synthetic lethality in tumors with homologous recombination deficiency. However, the efficacy of niraparib for rare subtypes of ovarian cancer remains unclear. In this study, we investigated the efficacy of niraparib using patient-derived xenograft (PDX) models with rare subtypes of ovarian cancer. Methods: We tried to establish PDXs by transplanting patients' tissue specimens subcutaneously into NOG mice. Immunohistochemical (IHC) staining for SLFN11(D-2 sc515071X, Santa Cruz), a possible efficacy biomarker of PARP inhibitor, and genetic analysis of 160 cancer-related genes (GeneRead Human Comprehensive Cancer Panel, Qiagen) were performed using the parental tumors. Response to carboplatin (CBDCA) and niraparib was analyzed using the PDX models. Results: A total of 3 PDXs with rare subtypes of ovarian cancer were established including carcinosarcoma (CS), adenocarcinoma (Adeno) with neuroendocrine carcinoma (NEC), and clear cell carcinoma (CCC). Histologically, PDX tumors generally mimicked their parental tumors. Positive staining for SLFN11 was observed in CS and Adeno with NEC, both of which had TP53 alterations, while only the CS case had BRCA2 pathogenic mutation. In the PDX of CS, both CBDCA and niraparib suppressed tumor growth in a dose-dependent manner. In the PDX of Adeno with NEC, CBDCA significantly suppressed tumor growth, while niraparib did not. Neither CBDCA nor niraparib inhibited tumor growth in the PDX of CCC without BRCA mutation.

Conclusion: The combination of SLFN11 expression in IHC and BRCA status may be an efficacy biomarker for niraparib in rare subtypes of ovarian cancer.

Poster (031)

Epithelial Ovarian Cancer including Borderline Tumor https://doi.org/10.3802/jgo.2021.32.S1.031

\section{High gradient magnetic separation of circulating endothelial cells as a diagnostic tool for ovarian cancer}

\section{Saranya Chanpanitkitchot,, 1,* Dumrong Mairiang, ${ }^{2}$ Prapat Suriyaphol $^{2}$}

'Rajavithi Hospital, Bangkok, Thailand (saranya.jibi@gmail.com)

${ }^{2}$ Faculty of Medicine Siriraj Hospital, Mahidol University, Bangkok, Thailand 
Objective: This study aims to evaluate circulating tumor cells isolated by high gradient magnetic separation of circulating endothelial cells (CECs) as a diagnostic tool for ovarian cancer. Methods: Thirty-six newly diagnosis with pelvic mass who were scheduled for surgery were included in this study. Blood samples were obtained from patients. CECs were identified with fluorescent microscopy by expert cytologists. The diagnostic performance of CECs was compared with the histopathological results.

Results: Definite diagnosis showed that twenty-six patients were later diagnosed as having malignant ovarian cancer (69.4\%), 5 cancerous patients (19.2\%) had metastatic ovarian tumor from gastrointestinal origin, and 10 patients were diagnosed as benign ovarian tumor (30.6\%). Serum from patients were extracted for CECs were found in 10 patients. CECs demonstrated modest diagnostic performance for malignant ovarian cancer with accuracy, sensitivity and specificity of $38.9 \%, 26.9 \%$, and $70.0 \%$, respectively.

Conclusion: CECs has low sensitivity. However, the clinical value of CECs in borderline ovarian tumor cases must be further investigated. This technique needs more development to improve sensitivity for diagnostic for ovarian cancer.

Poster (032)

Epithelial Ovarian Cancer including Borderline Tumor

https://doi.org/10.3802/jgo.2021.32.S1.032

\section{Knockdown of E2F4 suppresses the growth of ovarian cancer cells via cell cycle pathway}

\section{Young Tae Kim,," Eun Ji Nam, ${ }^{1}$ Sang Wun Kim,' Dae Woo Lee \\ 'Yonsei University College of Medicine, Seoul, Korea (ytkchoi@yuhs.ac) ${ }^{2}$ Catholic University College of Medicine, Seoul, Korea}

Objective: Ovarian cancer is one of the leading causes of death from gynecological cancer in the developed countries. The E2F family is a group of transcription factors that play decisive roles in the control of cell proliferation and cell cycle progression through the transcriptional activation of target genes in higher eukaryotes. However, the carcinogenic role of E2F transcription factor 4 (E2F4) remains unclear in ovarian cancer. In this study, we investigated the underlying molecular mechanism of E2F4 in human ovarian cancer cells (OCC).

Methods: The expression levels of E2F4 were demonstrated by quantitative real-time polymerase chain reaction (qRT-PCR). Small interfering RNA was used to suppress E2F4 expression. The effects of suppressing E2F4 on cell proliferation, migration and differentiation were evaluated by cell proliferation assay, wound healing assay and colony formation assay in vitro. The expression level of cyclins in OCC influenced by E2F4 was detected by Western blot.

Results: The expression of E2F4 mRNA and protein showed a significant higher level in OCC lines compared with controls. Depletion of E2F4 inhibited the cell proliferation and suppressed the cell migration and colony formation ability compared to control. Moreover, Western blot analysis showed that the expression of cyclin A, cyclin D and CDK2 was upregulated after the knockdown of E2F4.

Conclusion: Our study was consistent with the concept that E2F4 might contributed to proliferation and migration of OCCs by regulating the expression of cell cycle component in epithelial ovarian cancer.

Poster (O33)

Epithelial Ovarian Cancer including Borderline Tumor https://doi.org/10.3802/jgo.2021.32.\$1.033

\section{Multimodality active prehabilitation in patients undergoing surgery for epithelial ovarian cancer: a tertiary cancer center experience from Eastern India}

\author{
Upasana Palo," Jaydip Bhaumik, Anik Ghosh, Debapriya Mondal, \\ Jyoti B Lama, Zakaa Zawuaa
}

Tata Medical Center, Kolkata, India (upasana.obg@gmail.com)

Objective: This study aimed to determine if a multimodality active prehabilitation (MAP) program improves postoperative outcomes in patients undergoing surgery for epithelial ovarian cancer (EOC).

Methods: This was a prospective observational study over 6 months including 52 patients of EOC who underwent cytoreductive surgeries after MAP. These patients were compared with 51 patients with similar clinical characteristics at the same institution in the 6 months preceding the implementation of a MAP program (non-MAP group) by comparing frequency of complications with Clavien-Dindo (CD) score, median length of hospital stay, median time to chemotherapy, and 30-day-readmission rates.

Results: Twenty-nine patients (55.8\%) with interval debulking surgery, 13 with interval debulking surgery-hyperthermic intraperitoneal chemotherapy (25.0\%), 6 (11.5\%) with primary debulking surgery, 3 (5.8\%) with completion surgery, and 1 patient $(1.9 \%)$ with secondary cytoreduction were included in the MAP group ( $n=52$ ). $C D \geq 3$ complications were similar in the 2 groups. Median length of hospital stay was significantly shorter in the MAP group compared to the non-MAP group ( 8 vs. 10 days, $\mathrm{p}=0.006$ ). Median time to chemotherapy was significantly reduced ( 26 days vs. 30 days, $p=0.007$ ) and 30 -day- 